\title{
Abusing a hypergraph partitioner for unweighted graph partitioning
}

\author{
B. O. Fagginger Auer and R. H. Bisseling
}

Mathematics Institute, Utrecht University, Budapestlaan 6, 3584 CD, Utrecht, the Netherlands

B.O.FaggingerAuer@uu.nl

R.H.Bisseling@uu.nl

\begin{abstract}
We investigate using the Mondriaan matrix partitioner for unweighted graph partitioning in the communication volume and edgecut metrics. By converting the unweighted graphs to appropriate matrices, we measure Mondriaan's performance as a graph partitioner for the 10th DIMACS challenge on graph partitioning and clustering. We find that Mondriaan can effectively be used as a graph partitioner: w.r.t. the edge-cut metric, Mondriaan's average results are within $21 \%$ of the best known results as listed in Chris Walshaw's partitioning archive.
\end{abstract}

\section{Introduction}

In this paper, we use the Mondriaan matrix partitioner [21] to partition the graphs from the 10th DIMACS challenge on graph partitioning and clustering [1]. In this way, we can compare Mondriaan's performance as a graph partitioner with the performance of the state-of-the-art partitioners participating in the challenge.

An undirected graph $G$ is a pair $(V, E)$, with vertices $V$, and edges $E$ that are of the form $\{u, v\}$ for $u, v \in V$ with possibly $u=v$. For vertices $v \in V$, we denote the set of all of $v$ 's neighbours by

$$
V_{v}:=\{u \in V \mid\{u, v\} \in E\} .
$$

Note that vertex $v$ is a neighbour of itself precisely when the self-edge $\{v, v\} \in E$.

Hypergraphs are a generalisation of undirected graphs, where edges can contain an arbitrary number of vertices. A hypergraph $\mathcal{G}$ is a pair $(\mathcal{V}, \mathcal{N})$, with vertices $\mathcal{V}$, and nets (or hyperedges) $\mathcal{N}$; nets are subsets of $\mathcal{V}$ that can contain any number of vertices.

Let $\epsilon>0, k \in \mathbf{N}$, and $G=(V, E)$ be an undirected graph. Then a valid solution to the graph partitioning problem for partitioning $G$ into $k$ parts with imbalance $\epsilon$, is a partitioning $\Pi: V \rightarrow\{1, \ldots, k\}$ of the graph's vertices into $k$ parts, each part $\Pi^{-1}(\{i\})$ containing at most

$$
\left|\Pi^{-1}(\{i\})\right| \leq(1+\epsilon)\left\lceil\frac{|V|}{k}\right\rceil, \quad(1 \leq i \leq k)
$$


vertices.

To measure the quality of a valid partitioning we use two different metrics. The communication volume metric ${ }^{1}[1]$ is defined by

$$
\mathrm{CV}(\Pi):=\max _{1 \leq i \leq k} \sum_{\substack{v \in V \\ \Pi(v)=i}}\left|\Pi\left(V_{v}\right) \backslash\{\Pi(v)\}\right| .
$$

For each vertex $v$, we determine the number of different parts $\pi(v)$ in which $v$ has neighbours, except $\Pi(v)$. Then, the communication volume is given by the maximum over $i$, of the sum of all $\pi(v)$ for vertices $v$ belonging to part $i$.

The edge-cut metric [1], defined as

$$
\operatorname{EC}(\Pi):=|\{\{u, v\} \in E \mid \Pi(u) \neq \Pi(v)\}|,
$$

measures the number of edges that exist between different parts of the partitioning $\Pi$.

\begin{tabular}{|lr|l|l|}
\hline Name & Ref. & $\begin{array}{l}\text { Graph/ } \\
\text { hypergraph }\end{array}$ & $\begin{array}{l}\text { Sequential/ } \\
\text { parallel }\end{array}$ \\
\hline Chaco & {$[13]$} & graph & sequential \\
Metis & {$[14]$} & graph & sequential \\
Scotch & {$[18]$} & graph & sequential \\
Jostle & {$[22]$} & graph & parallel \\
ParMetis & {$[16]$} & graph & parallel \\
PT-Scotch & {$[10]$} & graph & parallel \\
hMETIS & {$[15]$} & hypergraph & sequential \\
ML-Part & {$[6]$} & hypergraph & sequential \\
Mondriaan & {$[21]$} & hypergraph & sequential \\
PaToH & {$[8]$} & hypergraph & sequential \\
Parkway & {$[20]$} & hypergraph & parallel \\
Zoltan & {$[12]$} & hypergraph & parallel \\
\hline
\end{tabular}

Table 1. Overview of available software for partitioning (hyper)graphs from [2].

There exist a lot of different (hyper)graph partitioners, which are summarised in Table 1 from [2]. All partitioners follow a multi-level strategy [5], where the (hyper)graph is coarsened by generating a matching of the (hyper)graph's vertices and contracting matched vertices to a single vertex. Doing this recursively creates a hierarchy of increasingly coarser approximations of the original (hyper)graph. After this has been done, an initial partitioning is generated on the coarsest (hyper)graph in the hierarchy, i.e. the one possessing the smallest number of vertices. This partitioning is subsequently propagated to the finer (hyper)graphs in the hierarchy and refined at each level (e.g. using the KernighanLin algorithm [17]), until we reach the original (hyper)graph and obtain the final partitioning.

${ }^{1}$ We forgo custom edge and vertex weights and assume they are all equal to one, because Mondriaan's hypergraph partitioner does not support net weights. 


\section{Mondriaan}

Mondriaan has been designed to partition the matrix and the vectors for a parallel sparse matrix-vector multiplication, where a sparse matrix $A$ is multiplied by a dense input vector $\mathbf{v}$ to give a dense output vector $\mathbf{u}=A \mathbf{v}$ as the result. First, the matrix partitioning algorithm is executed to minimise the total communication volume $\mathrm{LV}(\Pi)$ of the partitioning, defined below, and then the vector partitioning algorithm is executed with the aim of balancing the communication among the processors. The matrix partitioning itself does not aim to achieve such balance, but it is not biased in favour of any processor part either.

\begin{tabular}{|lr|c|l|}
\hline Name & Ref. & $\mathcal{V}$ & \multicolumn{1}{|c|}{$\mathcal{N}$} \\
\hline Column-net & {$[7]$} & $\left\{r_{1}, \ldots, r_{m}\right\}$ & $\left\{\left\{r_{i} \mid 1 \leq i \leq m, a_{i j} \neq 0\right\} \mid 1 \leq j \leq n\right\}$ \\
\hline Row-net & {$[7]$} & $\left\{c_{1}, \ldots, c_{n}\right\}$ & $\left\{\left\{c_{j} \mid 1 \leq j \leq n, a_{i j} \neq 0\right\} \mid 1 \leq i \leq m\right\}$ \\
\hline Fine-grain & {$[9]$} & $\left\{v_{i j} \mid a_{i j} \neq 0\right\}$ & $\underbrace{\left\{\left\{v_{i j} \mid 1 \leq i \leq m, a_{i j} \neq 0\right\} \mid 1 \leq j \leq n\right\}}_{\text {column nets }}$ \\
& & & $\underbrace{\left\{\left\{v_{i j} \mid 1 \leq j \leq n, a_{i j} \neq 0\right\} \mid 1 \leq i \leq m\right\}}_{\text {row nets }}$ \\
\hline
\end{tabular}

Table 2. Available representations of an $m \times n$ matrix $A=\left(a_{i j}\right)$ by a hypergraph $\mathcal{G}=(\mathcal{V}, \mathcal{N})$ in Mondriaan.

Mondriaan uses recursive bipartitioning to split the matrix or its submatrices repeatedly into two parts, choosing the best of the row or column direction in the matrix. The current submatrix is translated into a hypergraph by the columnnet or row-net model, respectively (see Table 2). Another possibility is to split the submatrix based on the fine-grain model, and if desired the best split of the three methods can be chosen. The outcome of running Mondriaan is a twodimensional partitioning of the sparse matrix (i.e., a partitioning where both the matrix rows and columns are split). The number of parts is not restricted to a power of two, as Mondriaan can split parts according to a given ratio such as $2: 1$. After each split, Mondriaan adjusts the weight balancing goals of the new parts obtained, as the new part that receives the largest fraction of the weight will need to be stricter in allowing an imbalance during further splits than the part with the smaller fraction.

If the input vector and output vector can be partitioned independently, the vector partitioning algorithm usually has enough freedom to achieve a reasonable communication balancing. If the matrix is square, and both vectors must be partitioned in the same way, then there is usually little freedom. Sometimes, the total communication volume must even be increased because of the identical vector partitioning. If the matrix diagonal has only nonzero elements, however, the vector partitioning can be achieved without incurring additional communication by assigning vector components $v_{i}$ and $u_{i}$ to the same processor as the diagonal matrix element $a_{i i}$. More details on the matrix and vector partitioning 
can be found in [21]; improved methods for vector partitioning are given in [4], see also [3].

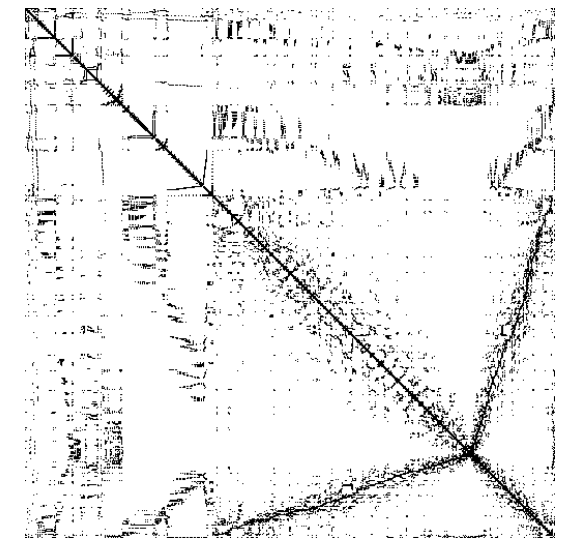

(a) $k=1$

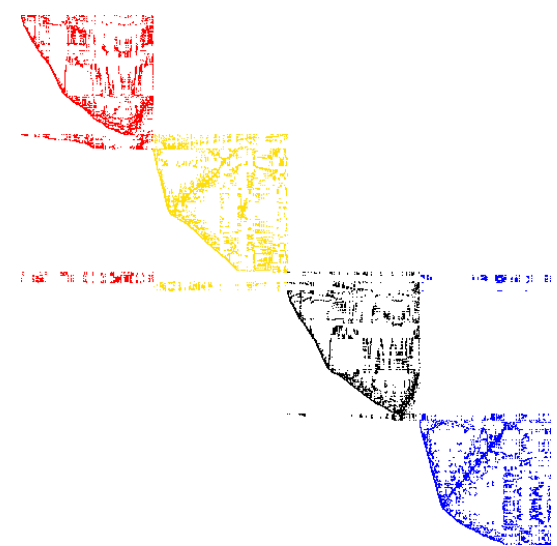

(c) $k=4$

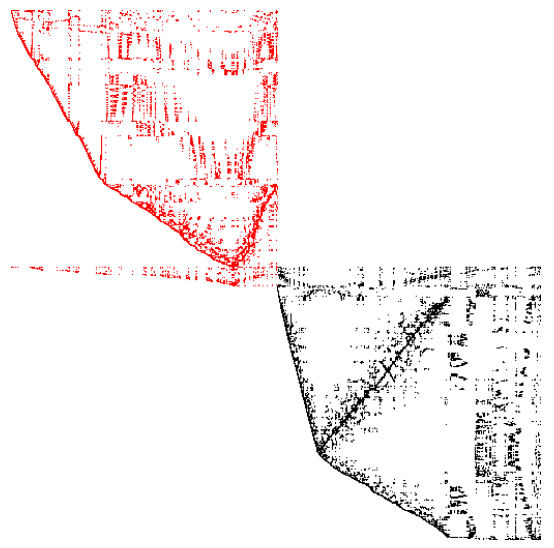

(b) $k=2$

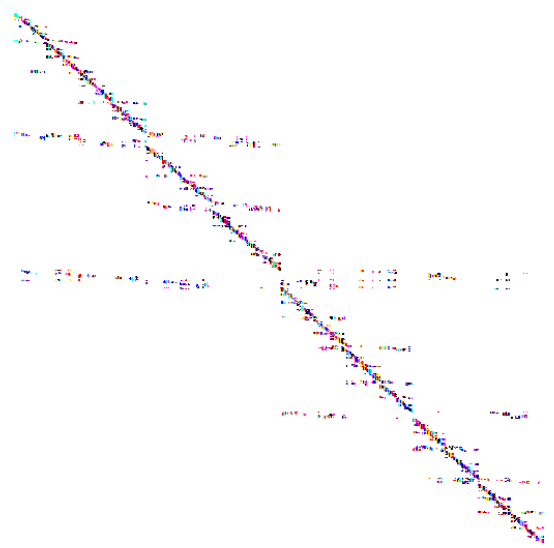

(d) $k=1024$

Fig. 1. Mondriaan 1D column partitioning of the graph fe_tooth, modelled as a sparse matrix cf. Thm. 1 , into $k=1,2,4,1024$ parts with imbalance $\epsilon=0.03$. The rows and columns of the matrices have been permuted for $k>1$ to Separated Block Diagonal form, see [23].

Here, we will use Mondriaan as a hypergraph partitioner, which can be done by choosing the column direction in all splits, so that columns are vertices and rows are nets. This means that we use Mondriaan in one-dimensional mode, as only rows will be split. Fig. 1 illustrates this splitting procedure. Mondriaan has 
the option to use its own, native hypergraph bipartitioner, or link to the external partitioner $\mathrm{PaToH}$ [8]. In the present work, we use the native partitioner.

For the graph partitioning challenge posed by DIMACS, we try to fit the existing software to the aims of the challenge. One could say that this entails abusing the software, as it was designed for a different purpose, namely matrix and hypergraph partitioning. Using a hypergraph partitioner to partition graphs will be at the cost of some additional, unnecessary overhead. Still, it will be interesting to see how the Mondriaan software performs in this unforeseen mode, and to compare the quality of the generated partitionings to the quality of partitionings generated by other software, in particular by graph partitioning packages.

In the situation of the challenge, we can only use the matrix partitioning of Mondriaan and not the vector partitioning, as the vertex partitioning of the graph is already completely determined by the column partitioning of the matrix. The balance of the communication will then solely depend on the balance achieved by the matrix partitioning.

Internally, Mondriaan's hypergraph partitioner solves the following problem. For a hypergraph $\mathcal{G}=(\mathcal{V}, \mathcal{N})$ with vertex weights $\zeta: \mathcal{V} \rightarrow \mathbf{N}$, an imbalance factor $\epsilon>0$, and a number of parts $k \in \mathbf{N}$, Mondriaan's partitioner produces a partitioning $\Pi: \mathcal{V} \rightarrow\{1, \ldots, k\}$ such that

$$
\zeta\left(\Pi^{-1}(\{i\})\right) \leq(1+\epsilon)\left\lceil\frac{\zeta(\mathcal{V})}{k}\right\rceil, \quad(1 \leq i \leq k)
$$

where the partitioner tries to minimise the $(\lambda-1)$-volume

$$
\mathrm{LV}(\Pi):=\sum_{n \in \mathcal{N}}(|\Pi(n)|-1)
$$

We will now translate the DIMACS partitioning problems from Sec. 1 to the hypergraph partitioning problem that Mondriaan is designed to solve, by creating a suitable hypergraph $\mathcal{G}$, encoded as a sparse matrix $A$ in the row-net model.

\subsection{Minimising communication volume}

Let $G=(V, E)$ be a given graph, $k \in \mathbf{N}$, and $\epsilon>0$. Our aim will be to construct a matrix $A$ from $G$ such that minimising eq. (5) subject to eq. (4) enforces minimisation of eq. (2) subject to eq. (1).

To satisfy eq. (1), we need to create one column in $A$ for each vertex in $V$, such that the hypergraph represented by $A$ in the row-net model will have $\mathcal{V}=V$. This is also necessary to have a direct correspondence between partitionings of the vertices $V$ of the graph and the vertices $\mathcal{V}$ of the hypergraph. Setting the weights $\zeta$ of all vertices/matrix columns to 1 will then ensure that eq. (1) is satisfied if and only if eq. (4) is satisfied. 
It is a little more tricky to match eq. (2) to eq. (5). Note that because of the maximum in eq. (2), we are not able to create an equivalent formulation. However, as

$$
\mathrm{CV}(\Pi) \leq \sum_{i=1}^{k} \sum_{\substack{v \in V \\ \Pi(v)=i}}\left|\Pi\left(V_{v}\right) \backslash\{\Pi(v)\}\right|=\sum_{v \in V}\left|\Pi\left(V_{v}\right) \backslash\{\Pi(v)\}\right|,
$$

we can provide an upper bound, which we can use to limit $\mathrm{CV}(\Pi)$. We need to choose the rows of $A$, corresponding to nets in the row-net hypergraph $\mathcal{G}=$ $(\mathcal{V}, \mathcal{N})$, such that eq. (6) and eq. (5) are in agreement.

For a net $n \in \mathcal{N}$, we have that $n \subseteq \mathcal{V}=V$ is simply a collection of vertices of $G$, so $|\Pi(n)|$ in eq. (5) equals the number of different parts in which the vertices of $n$ are contained. In eq. (6) we count, for a vertex $v \in V$, all parts in which $v$ has a neighbour, except $\Pi(v)$. Note that this number equals $\left|\Pi\left(V_{v}\right) \backslash\{\Pi(v)\}\right|=$ $\left|\Pi\left(V_{v} \cup\{v\}\right)\right|-1$.

Hence, we should pick $\mathcal{N}:=\left\{V_{v} \cup\{v\} \mid v \in V\right\}$ as the set of nets, for eq. (6) and eq. (5) to agree. In the row-net matrix model, this corresponds to letting $A$ be a matrix with a row for every vertex $v \in V$, filled with nonzeros $a_{v} v$ and $a_{v w}$ for all $w \in V_{v} \backslash\{v\}$. Then, for this hypergraph $\mathcal{G}$, we have by eq. (6) that $\mathrm{CV}(\Pi) \leq \operatorname{LV}(\Pi)$. Note that since the communication volume is defined as a maximum, we also have that $k \mathrm{CV}(\Pi) \geq \operatorname{LV}(\Pi)$.

Theorem 1. Let $G=(V, E)$ be a given graph, $k \in \mathbf{N}$, and $\epsilon>0$. Let $A$ be the $|V| \times|V|$ matrix with entries

$$
a_{v w}:= \begin{cases}1 & \text { if }\{v, w\} \in E \text { or } v=w, \\ 0 & \text { otherwise, }\end{cases}
$$

for $v, w \in V$, and $\mathcal{G}=(\mathcal{V}, \mathcal{N})$ the hypergraph corresponding to $A$ in the row-net model with vertex weights $\zeta(v)=1$ for all $v \in \mathcal{V}$.

Then, for every partitioning $\Pi: V \rightarrow\{1, \ldots, k\}$, we have that $\Pi$ satisfies eq. (1) if and only if $\Pi$ satisfies eq. (4), and

$$
\frac{1}{k} L V(\Pi) \leq C V(\Pi) \leq L V(\Pi)
$$

\subsection{Minimising edge cut}

We will now follow the same procedure as in Sec. 2.1 to construct a matrix $A$ such that minimising eq. (5) subject to eq. (4) is equivalent to minimising eq. (3) subject to eq. (1).

As in Sec. 2.1, the columns of $A$ should correspond to the vertices $V$ of $G$ to ensure that eq. (4) is equivalent to eq. (1).

Eq. (3) simply counts all of $G$ 's edges that contain vertices belonging to two parts of the partitioning $\Pi$. Since every edge contains vertices belonging to at least one part, and at most two parts, this yields

$$
\mathrm{EC}(\Pi)=\sum_{e \in E}(|\Pi(e)|-1)
$$


Choosing $\mathcal{N}:=E$ will therefore give us a direct correspondence between eq. (5) and eq. (3).

Theorem 2. Let $G=(V, E)$ be a given graph, $k \in \mathbf{N}$, and $\epsilon>0$. Let $A$ be the $|E| \times|V|$ matrix with entries

$$
a_{e v}:= \begin{cases}1 & \text { if } v \in e, \\ 0 & \text { otherwise, }\end{cases}
$$

for $e \in E, v \in V$, and $\mathcal{G}=(\mathcal{V}, \mathcal{N})$ the hypergraph corresponding to $A$ in the row-net model with vertex weights $\zeta(v)=1$ for all $v \in \mathcal{V}$.

Then, for every partitioning $\Pi: V \rightarrow\{1, \ldots, k\}$, we have that $\Pi$ satisfies eq. (1) if and only if $\Pi$ satisfies eq. (4), and

$$
E C(\Pi)=L V(\Pi) .
$$

With Thm. 1 and Thm. 2, we know how to translate a given graph $G$ to a hypergraph that Mondriaan can partition to obtain solutions to the DIMACS partitioning challenges.

\section{Results}

We measure Mondriaan's performance as a graph partitioner by partitioning graphs from the walshaw/ [19] and matrix/ [11] categories of the DIMACS test bed [1], see Table 3. This is done by converting the graphs to matrices, as expressed by Thm. 1 and Thm. 2, and partitioning these matrices with an updated version of Mondriaan 3.11, using the onedimcol splitting strategy (since the matrices represent row-net hypergraphs) with the lambda1 metric (cf. eq. (5)). The imbalance is set to $\epsilon=0.03$, the number of parts to $k=2,4, \ldots, 1024$, and we average the recorded communication volumes and edge cuts over 10 (walshaw/) or 5 (matrix/) runs (as Mondriaan uses random tie-breaking) of the Mondriaan partitioner. Note that we did not take the best result of the set of runs, as we are interested in the average performance of Mondriaan. All results were recorded on a dual quad-core AMD Opteron 2378 system with $32 \mathrm{GiB}$ of main memory and can be found in Tables $4-6$ and Figures 2 and 3 .

Results for graphs from the walshaw/ category for the edge-cut metric, Table 5 , can directly be compared with the best known partitionings with $3 \%$ imbalance from http://staffweb.cms.gre.ac.uk/ wc06/partition/ [19]. Compared to the results retrieved on November 2, 2011, we find that Mondriaan performs rather well, except for the graph add32. If we take the average of the relative edge cuts over all graphs in walshaw/ and all values $k=2,4, \ldots, 64$, then Mondriaan performs $21 \%$ worse than the best results from [19], and only $16 \%$ worse if add32 is excluded. It should be noted that we compare the average edge cut obtained by Mondriaan to the best known edge cuts from [19]. 


\begin{tabular}{|l|r|r|}
\hline$G$ & $|V|$ & $|E|$ \\
\hline add20 & 2,395 & 7,462 \\
data & 2,851 & 15,093 \\
3elt & 4,720 & 13,722 \\
uk & 4,824 & 6,837 \\
add32 & 4,960 & 9,462 \\
bcsstk33 & 8,738 & 291,583 \\
whitaker3 & 9,800 & 28,989 \\
crack & 10,240 & 30,380 \\
wing_nodal & 10,937 & 75,488 \\
fe_4elt2 & 11,143 & 32,818 \\
vibrobox & 12,328 & 165,250 \\
bcsstk29 & 13,992 & 302,748 \\
4elt & 15,606 & 45,878 \\
fe_sphere & 16,386 & 49,152 \\
cti & 16,840 & 48,232 \\
memplus & 17,758 & 54,196 \\
cs4 & 22,499 & 43,858 \\
bcsstk30 & 28,924 & $1,007,284$ \\
bcsstk31 & 35,588 & 572,914 \\
fe_pwt & 36,519 & 144,794 \\
bcsstk32 & 44,609 & 985,046 \\
fe_body & 45,087 & 163,734 \\
t60k & 60,005 & 89,440 \\
wing & 62,032 & 121,544 \\
brack2 & 62,631 & 366,559 \\
finan512 & 74,752 & 261,120 \\
fe_tooth & 78,136 & 452,591 \\
fe_rotor & 99,617 & 662,431 \\
598a & 110,971 & 741,934 \\
fe_ocean & 143,437 & 409,593 \\
144 & 144,649 & $1,074,393$ \\
wave & 156,317 & $1,059,331$ \\
m14b & 214,765 & $1,679,018$ \\
auto & 448,695 & $3,314,611$ \\
\hline
\end{tabular}

\begin{tabular}{|l|r|r|}
\hline$G$ & $|V|$ & $|E|$ \\
\hline af_shel19 & 504,855 & $8,542,010$ \\
audikw1 & 943,695 & $38,354,076$ \\
ldoor & 952,203 & $22,785,136$ \\
ecology2 & 999,999 & $1,997,996$ \\
ecology1 & $1,000,000$ & $1,998,000$ \\
thermal2 & $1,227,087$ & $3,676,134$ \\
af_shel110 & $1,508,065$ & $25,582,130$ \\
G3_circuit & $1,585,478$ & $3,037,674$ \\
kkt_power & $2,063,494$ & $6,482,320$ \\
nlpkkt120 & $3,542,400$ & $46,651,696$ \\
cage15 & $5,154,859$ & $47,022,346$ \\
nlpkkt160 & $8,345,600$ & $110,586,256$ \\
nlpkkt200 & $16,240,000$ & $215,992,816$ \\
\hline
\end{tabular}

Table 3. Graphs $G=(V, E)$ from the 10th DIMACS challenge [1] from the walshaw/ (left) and matrix/ (right) categories. 


\begin{tabular}{|c|c|c|c|c|c|c|c|c|c|c|}
\hline$G$ & 2 & 4 & 8 & 16 & 32 & 64 & 128 & 256 & 12 & 1024 \\
\hline $\operatorname{add} 20$ & 80 & 116 & 142 & 163 & 208 & & & & & \\
\hline ta & 66 & 92 & 5 & 7 & 74 & & & & & \\
\hline & 47 & 68 & 69 & 74 & 60 & 87 & & & & \\
\hline & 21 & 32 & 42 & 40 & 34 & 26 & & & & \\
\hline & 16 & 32 & 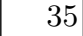 & 29 & 27 & 27 & & & & \\
\hline st & 494 & 734 & 96 & 817 & 35 & 495 & 384 & 375 & & \\
\hline$t$ & 5 & 2 & & & 32 & 68 & & & & \\
\hline$\sigma_{n}$ & 101 & 115 & 131 & 5 & 84 & 71 & 53 & 101 & 121 & \\
\hline ng_r & 460 & 688 & 564 & 494 & 395 & 273 & 194 & 150 & & \\
\hline 4 & 6 & 7 & & 3 & 4 & 91 & 52 & & & \\
\hline orc & 1,075 & 1,155 & 1,047 & 2 & 713 & 560 & 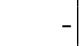 & 568 & & \\
\hline esst & 7 & 384 & 8 & 5 & 3 & 45 & 287 & & & \\
\hline $1 \mathrm{t}$ & & 5 & 106 & 106 & 8 & 81 & 64 & & & \\
\hline sp & & | & & & & 92 & 69 & 128 & & \\
\hline & 2 & 0 & 4 & 1 & 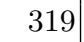 & 21 & 17 & 51 & & \\
\hline emp] & 2,608 & 1,850 & 1,113 & 792 & 732 & 22 & 61 & 692 & & \\
\hline 4 & 30 & 520 & 12 & 326 & 244 & 71 & 112 & 79 & 102 & \\
\hline SS & 7 & $6^{\prime}$ & 665 & 78 & 738 & 639 & 558 & 489 & 425 & \\
\hline SSt & 6 & 564 & 58 & 562 & | & 439 & 33 & 293 & 267 & \\
\hline pwt & 0 & 145 & 173 & 174 & 2 & 17 & 113 & 157 & 102 & \\
\hline & & 785 & & 794 & 629 & 05 & 89 & 349 & 298 & \\
\hline bod & 124 & 212 & 4 & 212 & 1 & 45 & 128 & 99 & 90 & 13 \\
\hline Ok & 74 & & 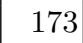 & 1 & 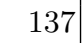 & 11 & 82 & 62 & 46 & \\
\hline$\gamma$ & 726 & 7 & 801 & 644 & 480 & 337 & 227 & 148 & 218 & \\
\hline brack & 238 & & & 739 & 619 & 99 & 98 & 309 & 259 & \\
\hline$f i$ & 94 & 123 & 155 & 156 & 88 & 175 & 17 & 206 & 188 & 14 \\
\hline & 1,299 & 1,393 & 1,429 & 1,224 & 946 & 807 & 582 & 398 & 275 & \\
\hline & & & & 1,297 & 1,028 & 813 & 640 & 445 & 379 & \\
\hline $598 a$ & 657 & 1,557 & & & 1,230 & 940 & 740 & 541 & 368 & 28 \\
\hline fe_oc & 274 & 918 & 72 & 1,184 & 971 & 714 & 514 & 354 & 242 & 16 \\
\hline & & 2,785 & 2, & 1,743 & 1,608 & 1,325 & 1,007 & 675 & 448 & $30:$ \\
\hline & & & 2,9 & 2,411 & 1,790 & 1,333 & 906 & 605 & 412 & 8 \\
\hline & & & & 2,234 & & & 1,087 & 827 & 583 & 39 \\
\hline ato & 2,580 & 5,059 & 5,177 & 4,406 & 3,287 & 2,581 & 1,775 & 1,182 & 800 & 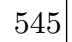 \\
\hline
\end{tabular}

Table 4. Average communication volume, eq. (2), over 10 Mondriaan runs, for graphs from the walshaw/ category, Table 3 , divided into $k=2,4, \ldots, 1024$ parts with imbalance $\epsilon=0.03$. A '-' indicates that Mondriaan was unable to generate a partitioning satisfying the balancing requirement, eq. (1). 


\begin{tabular}{|c|c|c|c|c|c|c|c|c|c|c|}
\hline$G$ & 2 & 4 & 8 & 16 & 32 & 64 & 128 & 256 & 512 & 1024 \\
\hline $\operatorname{add} 20$ & 701 & $\overline{1,239}$ & 1,860 & 2,328 & 2,742 & & & & & \\
\hline data & 210 & 428 & 770 & 1,303 & 2,080 & & & & & \\
\hline $3 e l t$ & 92 & 221 & 391 & 662 & 1,108 & 2,008 & - & - & & - \\
\hline uk & 22 & 53 & 113 & 190 & 318 & 544 & & & & . \\
\hline add32 & 48 & 109 & 201 & 298 & 493 & 800 & - & - & & - \\
\hline bcsstk 33 & 10,082 & 22,289 & 39,695 & 59,426 & 84,167 & 115,601 & 150,047 & 197,525 & & - \\
\hline whitaker3 & 130 & 395 & 731 & 1,213 & 1,879 & 2,815 & & & & \\
\hline crack & 197 & 407 & 767 & 1,223 & 1,915 & 2,846 & 4,211 & 8,861 & 27,546 & 28,206 \\
\hline wing_nodal & 1,750 & 3,864 & 6,078 & 9,206 & 13,142 & 17,653 & 23,074 & 30,739 & & \\
\hline fe_4elt2 & 130 & 356 & 658 & 1,140 & 1,842 & 2,866 & 4,144 & & & \\
\hline vibrobox & 11,456 & 21,559 & 31,780 & 39,980 & 48,792 & 56,222 & 70,224 & 100,660 & & - \\
\hline bcsstk29 & 3,009 & 8,719 & 17,760 & 27,286 & 41,233 & 61,076 & 98,161 & - & & - \\
\hline $4 \mathrm{elt}$ & 144 & 361 & 630 & 1,100 & 1,819 & 2,964 & 4,724 & & & \\
\hline fe_sphere & 426 & 844 & 1,308 & 2,006 & 2,902 & 4,173 & 5,918 & 11,041 & & \\
\hline cti & 346 & 1,011 & 1,882 & 3,075 & 4,400 & 6,136 & 8,479 & 12,184 & & \\
\hline memplus & 5,788 & 9,829 & 12,433 & 14,345 & 16,081 & 18,052 & 21,185 & 23,664 & & \\
\hline cs 4 & 402 & 1,082 & 1,717 & 2,484 & 3,448 & 4,688 & 6,234 & 8,267 & 15,904 & \\
\hline bcsstk30 & 6,483 & 17,522 & 38,673 & 80,951 & 128,679 & 189,268 & 272,306 & 381,846 & 527,735 & \\
\hline bcsstk31 & 2,880 & 7,935 & 15,805 & 27,376 & 44,942 & 67,652 & 98,515 & 140,666 & 199,171 & \\
\hline fe_pwt & 367 & 796 & 1,577 & 3,179 & 6,112 & 9,305 & 12,993 & 18,544 & 26,003 & - \\
\hline bcsstk32 & 5,657 & 11,674 & 25,134 & 43,940 & 69,459 & 105,490 & 154,161 & 225,289 & 317,952 & \\
\hline fe_body & 294 & 735 & 1,331 & 2,184 & 3,631 & 5,835 & 9,403 & 14,507 & 22,128 & 48,374 \\
\hline t60k & 82 & 259 & 548 & 994 & 1,632 & 2,547 & 3,813 & 5,565 & 8,152 & 12,087 \\
\hline wing & 912 & 1,921 & 2,966 & 4,551 & 6,628 & 9,044 & 12,010 & 15,890 & 24,206 & \\
\hline brack2 & 713 & 2,972 & 7,594 & 12,697 & 19,722 & 29,070 & 42,271 & 60,056 & 84,770 & \\
\hline finan512 & 162 & 510 & 1,125 & 1,872 & 2,896 & 11,089 & 22,030 & 39,294 & 57,481 & 75,316 \\
\hline fe_tooth & 4,140 & 7,892 & 13,284 & 20,226 & 28,577 & 39,601 & 53,141 & 71,917 & 96,280 & \\
\hline fe_rotor & 2,119 & 8,012 & 14,289 & 23,311 & 35,628 & 52,139 & 73,975 & 102,663 & 140,333 & \\
\hline $598 a$ & 2,457 & 8,343 & 17,031 & 28,841 & 44,104 & 63,806 & 88,039 & 118,654 & 157,227 & 209,672 \\
\hline fe_ocean & 329 & 1,918 & 4,720 & 8,832 & 14,401 & 22,074 & 30,787 & 42,182 & 57,226 & 75,811 \\
\hline 144 & 6,812 & 17,251 & 28,688 & 43,192 & 63,041 & 87,927 & 119,688 & 161,019 & 214,115 & 280,451 \\
\hline wave & 9,206 & 20,999 & 34,177 & 49,883 & 69,756 & 95,549 & 128,205 & 169,570 & 220,677 & 282,531 \\
\hline $\mathrm{m} 14 \mathrm{~b}$ & 3,973 & 13,688 & 27,861 & 48,599 & 75,484 & 109,861 & 154,290 & 212,062 & 286,225 & 381,217 \\
\hline auto & 10,364 & 28,026 & 52,424 & 89,759 & 134,990 & 193,265 & 267,282 & 360,509 & 476,470 & 621,578 \\
\hline
\end{tabular}

Table 5. Average edge cut, eq. (3), over 10 Mondriaan runs, for graphs from the walshaw/ category, Table 3 , divided into $k=2,4, \ldots, 1024$ parts with imbalance $\epsilon=$ 0.03. A '-' indicates that Mondriaan was unable to generate a partitioning satisfying the balancing requirement, eq. (1). 


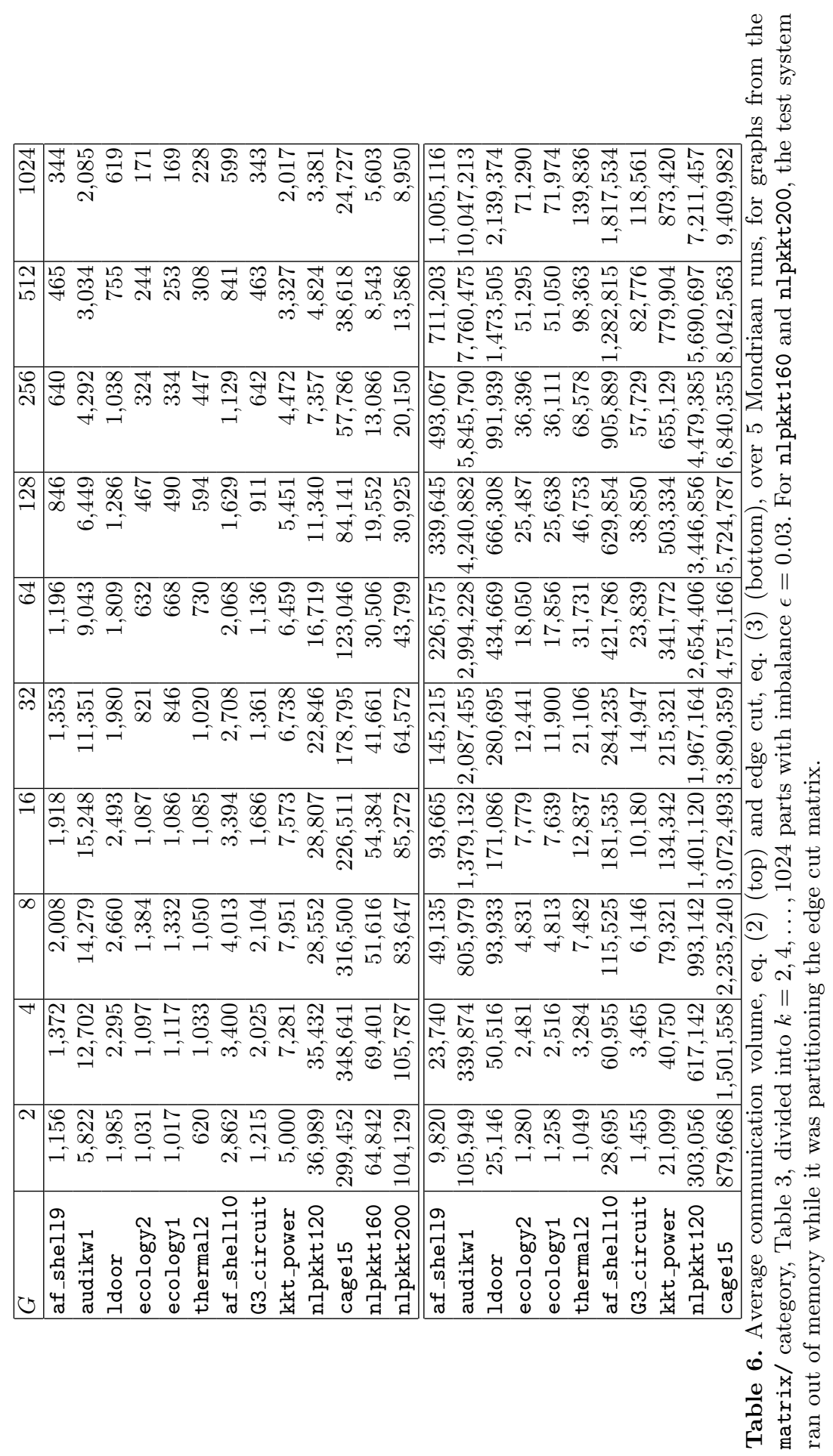


The strange dip in the communication volume for finan512 in Table 4 for $k=32$ parts can be explained by the fact that the graph $\mathrm{f}$ inan512 consists of 32 densely connected parts with few connections between them, see the visualisation of this graph in [11], such that there is a natural partitioning with very low communication volume in this case.

In Fig. 2, we plot the time required by Mondriaan to create a partitioning for both communication volume and edge cut. The number of nonzeros in the matrices from Thm. 1 and Thm. 2 equals $2|E|+|V|$ and $2|E|$, respectively. However, the matrix sizes are equal to $|V| \times|V|$ and $|E| \times|V|$, respectively. Therefore, even though the number of nonzeros in matrices from Thm. 2 is smaller, the larger number of nets (typically $|E|>|V|$, e.g. nlpkkt200) will lead to higher processing times and increased memory requirements for the edge-cut matrices, as can be seen when comparing Fig. 2(b) to Fig. 2(a).

We have also investigated the communication volume imbalance, defined for a partitioning $\Pi$ of $G$ into $k$ parts as

$$
\frac{\mathrm{CV}(\Pi)}{\mathrm{LV}(\Pi) / k}-1
$$

Eq. (9) measures the imbalance in communication volume and can be compared to the factor $\epsilon$ for vertex imbalance in eq. (1). We plot eq. (9) as a percentage for a selection of graphs in Fig. 3, where we see that the deviation of the communication volume $\mathrm{CV}(\Pi)$ from perfect balance, i.e. from $\operatorname{LV}(\Pi) / k$, is no more than $140 \%$ (for cage $15, k=1024$ ). Compared to the theoretical upper bound for the imbalance of $k-1$ (via eq. (7)), this is very good. This also means that at most a factor of 2.4 in communication volume per processor can still be gained by improving the communication balance. Therefore, as the number of parts increases, the different parts of the partitionings generated by Mondriaan are not only balanced in terms of vertices, cf. eq. (1), but also in terms of communication volume.

\section{Conclusion}

We have shown that it is possible to use the Mondriaan matrix partitioner as a graph partitioner by constructing appropriate matrices of a given graph for either the communication volume or edge-cut metric. Mondriaan's performance was measured by partitioning graphs from the 10th DIMACS challenge on graph partitioning and clustering, as well as comparing obtained edge cuts with the best known results from [19]: here Mondriaan's average edge cut was, on average, $21 \%$ higher than the best known. From these results we find that Mondriaan can effectively be used to perform graph partitioning.

To our surprise, the partitionings generated by Mondriaan are reasonably balanced in terms of communication volume, as shown in Fig. 3, even though Mondriaan does not perform explicit communication volume balancing during matrix partitioning. We attribute the observed balancing to the fact that the 


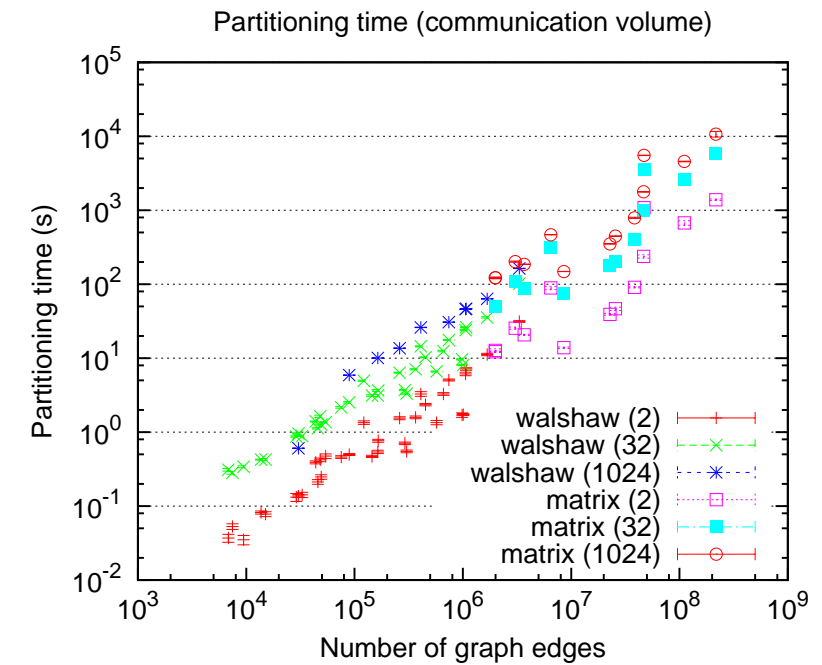

(a)

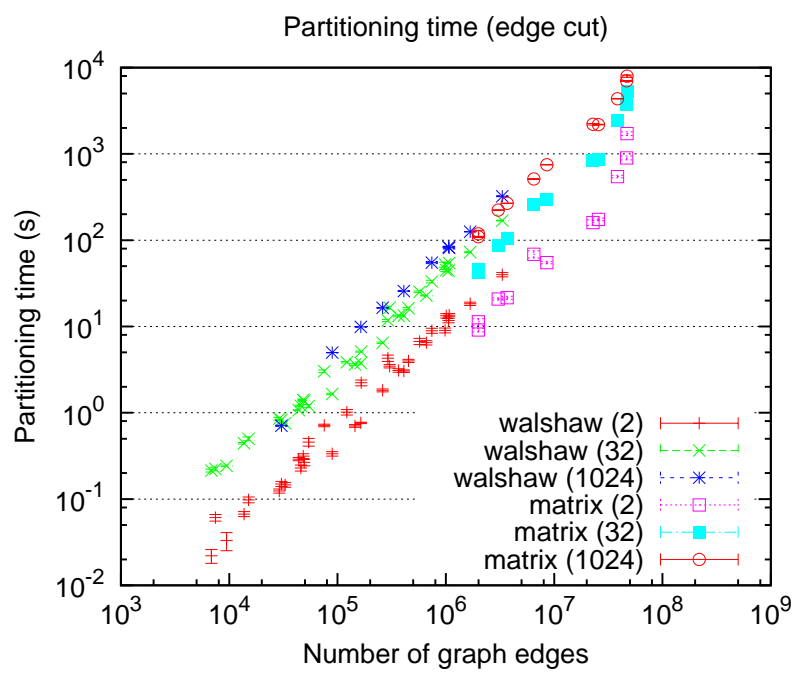

(b)

Fig. 2. The average partitioning time required by the Mondriaan partitioner to generate the partitionings from Table 4, 6, (a), and Table 5, 6, (b). 


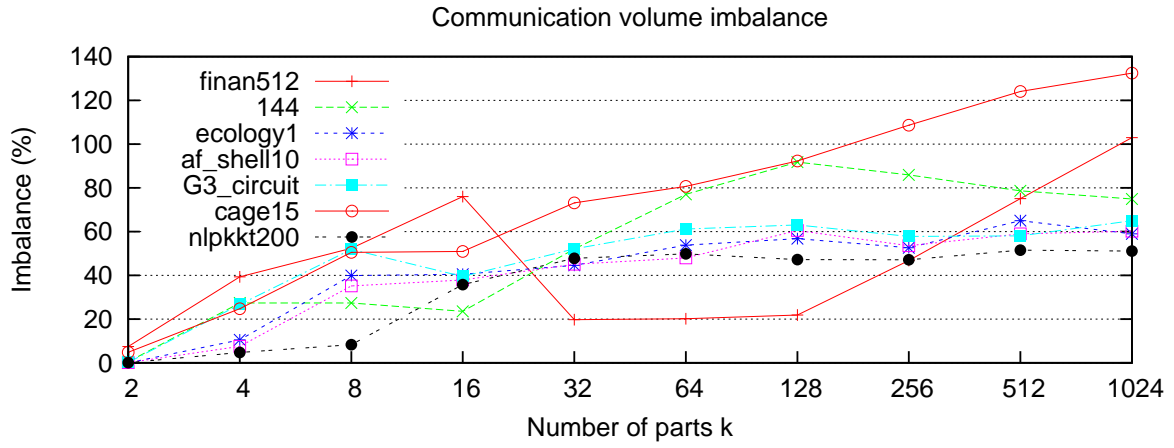

Fig. 3. The communication volume imbalance given by eq. (9), plotted as a percentage for several graphs.

Mondriaan algorithm performs random tie-breaking, without any preference for a specific part of the partitioning.

These tests also indicate the value of extending Mondriaan to take hypergraph net weights into account for the $(\lambda-1)$-metric, eq. $(5)$, because we could only perform unweighted graph partitioning due to the absence of this feature. We intend to add this feature in a next version of Mondriaan.

\section{References}

1. Bader, D.A., Sanders, P., Wagner, D., Meyerhenke, H., Hendrickson, B., Johnson, D.S., Walshaw, C., Mattson, T.G.: 10th DIMACS implementation challenge - graph partitioning and graph clustering (2012), http://www.cc.gatech.edu/dimacs10/ index.shtml

2. Bisseling, R.H., Fagginger Auer, B.O., Yzelman, A.N., van Leeuwen, T., Çatalyürek, Ü.V.: Two-dimensional approaches to sparse matrix partitioning. In: Naumann, U., Schenk, O. (eds.) Combinatorial Scientific Computing. CRC Press (2012)

3. Bisseling, R.H.: Parallel Scientific Computation: A Structured Approach using BSP and MPI. Oxford University Press, Oxford, UK (2004)

4. Bisseling, R.H., Meesen, W.: Communication balancing in parallel sparse matrixvector multiplication. Electronic Transactions on Numerical Analysis 21, 47-65 (2005), special Issue on Combinatorial Scientific Computing

5. Bui, T., Jones, C.: A heuristic for reducing fill-in in sparse matrix factorization. In: Proceedings Sixth SIAM Conference on Parallel Processing for Scientific Computing. pp. 445-452. SIAM, Philadelphia, PA (1993)

6. Caldwell, A.E., Kahng, A.B., Markov, I.L.: Improved algorithms for hypergraph bipartitioning. In: Proceedings Asia and South Pacific Design Automation Conference. pp. 661-666. ACM Press, New York (2000)

7. Çatalyürek, Ü.V., Aykanat, C.: Hypergraph-partitioning-based decomposition for parallel sparse-matrix vector multiplication. IEEE Transactions on Parallel and Distributed Systems 10(7), 673-693 (1999) 
8. Çatalyürek, Ü.V., Aykanat, C.: PaToH: A Multilevel Hypergraph Partitioning Tool, Version 3.0. Bilkent University, Department of Computer Engineering, Ankara, 06533 Turkey. PaToH is available at http://bmi.osu.edu/ umit/ software.htm (1999)

9. Çatalyürek, Ü.V., Aykanat, C.: A fine-grain hypergraph model for 2D decomposition of sparse matrices. In: Proceedings Eighth International Workshop on Solving Irregularly Structured Problems in Parallel (Irregular 2001). p. 118. IEEE Press, Los Alamitos, CA (2001)

10. Chevalier, C., Pellegrini, F.: PT-Scotch: a tool for efficient parallel graph ordering. Parallel Computing 34(6-8), 318-331 (2008)

11. Davis, T.A., Hu, Y.: The university of florida sparse matrix collection. ACM Transactions on Mathematical Software 38(1), 1-25 (2011)

12. Devine, K.D., Boman, E.G., Heaphy, R.T., Bisseling, R.H., Catalyurek, U.V.: Parallel hypergraph partitioning for scientific computing. In: Proceedings IEEE International Parallel and Distributed Processing Symposium 2006. IEEE Press (2006)

13. Hendrickson, B., Leland, R.: An improved spectral graph partitioning algorithm for mapping parallel computations. SIAM Journal on Scientific Computing 16(2), 452-469 (1995)

14. Karypis, G., Kumar, V.: A fast and high quality multilevel scheme for partitioning irregular graphs. SIAM Journal on Scientific Computing 20(1), 359-392 (1998)

15. Karypis, G., Kumar, V.: Multilevel k-way hypergraph partitioning. In: Proceedings 36th ACM/IEEE Conference on Design Automation. pp. 343-348. ACM Press, New York (1999)

16. Karypis, G., Kumar, V.: Parallel multilevel $k$-way partitioning scheme for irregular graphs. SIAM Review 41(2), 278-300 (1999)

17. Kernighan, B.W., Lin, S.: An efficient heuristic procedure for partitioning graphs. Bell System Technical Journal 29, 291-307 (1970)

18. Pellegrini, F., Roman, J.: Scotch: A software package for static mapping by dual recursive bipartitioning of process and architecture graphs. In: Proceedings High Performance Computing and Networking Europe. Lecture Notes in Computer Science, vol. 1067, pp. 493-498. Springer (1996)

19. Soper, A.J., Walshaw, C., Cross, M.: A combined evolutionary search and multilevel optimisation approach to graph-partitioning. J. of Global Optimization 29, 225-241 (2004)

20. Trifunović, A., Knottenbelt, W.J.: Parallel multilevel algorithms for hypergraph partitioning. Journal of Parallel and Distributed Computing 68(5), 563-581 (2008)

21. Vastenhouw, B., Bisseling, R.H.: A two-dimensional data distribution method for parallel sparse matrix-vector multiplication. SIAM Review 47(1), 67-95 (2005)

22. Walshaw, C., Cross, M.: JOSTLE: Parallel Multilevel Graph-Partitioning Software - An Overview. In: Magoules, F. (ed.) Mesh Partitioning Techniques and Domain Decomposition Techniques, pp. 27-58. Civil-Comp Ltd. (2007)

23. Yzelman, A.N., Bisseling, R.H.: Cache-oblivious sparse matrix-vector multiplication by using sparse matrix partitioning methods. SIAM Journal on Scientific Computing 31(4), 3128-3154 (2009) 